\title{
The relationship between disordered pursuit and vestibulo-ocular reflex suppression
}

\author{
BR CHAMBERS, MA GRESTY \\ From the MRC Neuro-otology Unit, Institute of Neurology, National Hospital, Queen Square, London, UK
}

SUMMARY The performance of the smooth pursuit reflex and the ability to suppress the vestibuloocular reflex were assessed in 10 normal subjects and in patients with a variety of diseases of the central nervous system. Pursuit was measured as the maximum velocity of slow phase eye movement in response to a laser target moving sinusoidally at various frequencies up to $1 \mathrm{~Hz}$ and with amplitudes stepped up to $35^{\circ}$ peak. Suppression of the vestibulo-ocular reflex was assessed with subjects seated in a Barany chair rotating sinusoidally in yaw at matching frequencies. The breakpoint of vestibulo-ocular reflex suppression was defined as the peak velocity of oscillation at which nystagmus appeared on electro-oculographic recording as determined by the method of ascending and descending thresholds. For normal subjects, at all frequencies, the breakpoint of suppression corresponded closely with the peak velocity of pursuit at the corresponding frequency of target oscillation. In some patients pursuit and suppression were comparably impaired. In others either pursuit or suppression could be selectively impaired with the other function left intact. The results demonstrate that the mechanisms of pursuit and visual suppression of the vestibulo-ocular reflex have similar dynamics and share a common pathway at least to the level of the cerebellum. Thereafter, there is presumably an anatomical and functional dissociation of the signals mediating the two functions. The key area involved appears to be the flocculus for lesions of this structure alone cause impairment of both functions. The findings also indicate that the appropriate way to test smooth pursuit in relationship to suppression is to increase the amplitude of target oscillation until the peak slow eye movement velocity is determined for each frequency. The finding that increasing excursion increases maximum pursuit velocity supports the view that pursuit has an acceleration limit which is more critical in determining performance than velocity limitations. The results establish the assessment of vestibulo-ocular reflex suppression as a powerful test of the integrity of CNS function independent of its previous association with disordered pursuit.

The function of the vestibulo-ocular reflex (VOR) is to generate compensatory eye movements which stabilise fixation on stationary targets during active and passive head movements. During pursuit of moving targets by active head turning, the VOR must be over-ridden, otherwise it would tend to drive the eyes in the inappropriate direction. This process is termed vestibulo-ocular reflex suppression (VORS). Experiments on normal subjects have demonstrated that there is a strong similarity between the frequency

Address for reprint requests: Dr MA Gresty, National Hospital Queen Square, London WC1N 3BG, UK.

Received 6 March 1982 and in revised form 10 September 1982.

Accepted 30 September 1982

BRC was supported by an Alexander Pigott Wernher Fellowship. response and velocity limits of the pursuit reflex and of VORS ${ }^{1}$ which has led to the hypothesis that pursuit is responsible for suppression. The proposed mechanism holds that when VOR generates eye movements off target, slip of the target image on the retina stimulates a cancelling pursuit movement. The hypothesis has gained support from clinical studies which have shown that patients with neurological disorders in whom pursuit is defective seem to have roughly corresponding disorders of VORS. ${ }^{2} 3$ Nevertheless, these studies lacked any kind of systematic investigation of the dynamic range of frequency response and velocity limits of the relevant oculomotor functions in the patients studied.

Using bedside tests for VORS ${ }^{3}$ and pursuit we have gained the distinct impression that although in most patients the two tasks are executed equally well or poorly, there are some patients in whom a marked 
dissociation is evident. During formal discussion on visual-vestibular interaction at a recent international symposium, other clinicians commented on similar observations. ${ }^{4}$ The present study examines the relationship between smooth pursuit and VORS in normal subjects and in neurological patients with intact vestibular responses who had signs of disordered pursuit and VORS. Although a close relationship exists in normals and most patients, the demonstration of complete dissociation in some patients indicates that other factors must also be important.

\section{Patients and methods}

The patients were selected on the basis that clinical examination revealed a deficit of pursuit and/or impaired VORS. Routine recordings of nystagmus and eye movements were obtained using conventional DC electro-oculography (EOG). These included examination for nystagmus in darkness, full-field optokinetic stimulation, and assessment of vestibular function by impulsive rotational stopping stimuli. To avoid confusion, patients with spontaneous horizontal nystagmus in or close to the primary position of gaze were excluded. Similarly only patients with intact vestibular responses were included since misleading results might be obtained from any patients with absent vestibular function. The patients were investigated according to the protocol outlined below. Ten volunteers were also examined to provide normative data.

Smooth pursuit in the horizontal plane was measured using a moving laser target, projected by means of a mirror mounted on a scanning motor, onto a tangent screen 2 metres from the subject. Recordings were made of responses to sinusoidal target motion graduated in frequency steps from $0 \cdot 1$ to $1 \mathrm{~Hz}$. At each frequency the amplitude of the target trajectory was increased through $5,10,15,20$, 27.5 and 35 degrees. To ensure optimal performance, patients were allowed to practice beforehand, and frequent rest periods were given during testing. Pursuit tasks were ordered from less difficult to more difficult with constant encouragement provided throughout. Those patients with distance glasses wore them during testing. Hand measurements of slow phase pursuit eye velocity were taken from raw data records using a nomograph. Peak velocity through primary position was measured for each combination of frequency and amplitude of target motion. Performance was characterised by the highest velocity pursuit movements produced as the eyes passed through primary position. VORS was tested with the patient seated and comfortably restrained in a Barany chair powered by a $18 \mathrm{Nm}$ torque motor. A fixation light of the same intensity and colour as the laser pursuit target, and subtending a similar visual angle was mounted to the chair in front of the subject. Low level ambient illumination of the surrounding stationary environment was used since previous study has shown that this may slightly aid VORS.5 The vestibular chair was driven by a function generator producing a sinusoidal stimulus at the same frequencies as those used for the pursuit tasks. The subject was instructed to attempt to maintain fixation on the target during oscillation, and the peak velocity of sinusoidal rotation was increased until it was observed that the subject was unable to maintain fixation. This was evident when the EOG revealed a consistent well-formed modulating pattern of compensatory eye movements in response to chair rotation, with or without superimposed nystagmus quick phases. The chair velocity was then reduced and the precise threshold or breakpoint of VORS was obtained, by the method of ascending and descending threshold judgements. When the breakpoint had been determined the fixation target was switched off to ensure that there was a well-formed vestibular response in total darkness. Rest intervals were allowed between each measurement and the subjects were continually encouraged to remain alert and perform to the best of their capabilities.

\section{Results}

\section{A. NORMAL SUBJECTS}

The results from the 10 normal controls were pooled and tabulated. Table 1 relates the means and standard deviations of peak pursuit eye velocity to the frequency and amplitude of target oscillation. At lower frequencies there was a linear relationship between eye velocity and target velocity with approximately unity gain, but at $0 \cdot 2 \mathrm{~Hz}$ the maximum possible pursuit velocity was not determined because peak target velocity was dependent on the limits of amplitude of target oscillation. At higher frequencies the pursuit gain fell progressively away from unity as target velocity was increased. The normative data demonstrate that there is considerable individual variation in performance approaching the limits of

Table 1 Velocity of pursuit eye movements in 10 normal subjects

\begin{tabular}{|c|c|c|c|c|c|c|c|}
\hline \multirow{2}{*}{$\begin{array}{l}\text { Target } \\
\text { frequency } \\
(\mathrm{Hz})\end{array}$} & \multicolumn{6}{|c|}{ Peak amplitude of target displacement in degrees } & \multirow{2}{*}{$\begin{array}{l}\text { Peak target velocity } \\
\text { at maximum amplitudes } \\
\text { attained }(\% / \mathrm{sec})\end{array}$} \\
\hline & 5 & 10 & 15 & 20 & $27 \cdot 5$ & 35 & \\
\hline $\begin{array}{l}0 \cdot 2 \\
0 \cdot 4 \\
0 \cdot 6 \\
0 \cdot 8 \\
1 \cdot 0\end{array}$ & $\begin{array}{r}7 \cdot 4(1 \cdot 0) \\
12 \cdot 9(2 \cdot 0) \\
18 \cdot 7(3 \cdot 2) \\
24 \cdot 1(8 \cdot 1) \\
20 \cdot 3(7 \cdot 7)\end{array}$ & $\begin{array}{l}13 \cdot 6(1 \cdot 3) \\
25 \cdot 8(3 \cdot 2) \\
34 \cdot 9(5 \cdot 9) \\
32 \cdot 8(11 \cdot 6) \\
34 \cdot 9(19 \cdot 2)\end{array}$ & $\begin{array}{l}19 \cdot 3(2 \cdot 6) \\
35 \cdot 1(4 \cdot 3) \\
44 \cdot 9(11 \cdot 0) \\
42 \cdot 0(15 \cdot 8) \\
37 \cdot 0(26 \cdot 5)\end{array}$ & $\begin{array}{l}25 \cdot 6(2 \cdot 0) \\
47 \cdot 2(4 \cdot 6) \\
60 \cdot 0(16 \cdot 7) \\
52 \cdot 4(23 \cdot 9) \\
40 \cdot 0(32 \cdot 8)\end{array}$ & $\begin{array}{l}35 \cdot 2(4 \cdot 4) \\
66 \cdot 9(7 \cdot 1)\end{array}$ & $\begin{array}{l}46 \cdot 4(3 \cdot 7)^{*} \\
84 \cdot 3(7 \cdot 1)\end{array}$ & $\begin{array}{l}44 \text { at } 35^{\circ} \text { displacement } \\
88 \text { at } 35^{\circ} \text { displacement } \\
131 \text { at } 20^{\circ} \text { displacement } \\
176 \text { at } 20^{\circ} \text { displacement } \\
220 \text { at } 20^{\circ} \text { displacement }\end{array}$ \\
\hline
\end{tabular}

The tabulated values represent the means (one SD in parentheses) of pursuit eye velocity measured as the eyes pass through primary gaze. * Maximum velocity of pursuit could not be tested because of physical restrictions on target displacement. 
Table 2 Breakpoint for vestibulo-ocular reflex suppression in 10 normal subjects

\begin{tabular}{ll}
\hline $\begin{array}{l}\text { Frequency of } \\
\text { chair oscillation }\end{array}$ & $\begin{array}{l}\text { Velocity at which VORS fails } \\
\text { Degrees/s }\end{array}$ \\
\hline $0.2 \mathrm{~Hz}$. & $72.5(17 \cdot 6)$ \\
0.4 & $65 \cdot 8(17 \cdot 4)$ \\
0.6 & $56 \cdot 3(17 \cdot 9)$ \\
0.8 & $44 \cdot 5(15 \cdot 0)$ \\
1.0 & $31.0(7 \cdot 7)$ \\
\hline
\end{tabular}

One SD in parentheses.

pursuit. For example with a stimulus of $0 \cdot 8 \mathrm{~Hz}, 100^{\circ} / \mathrm{s}$, the range of pursuit velocity was from 16 to $94^{\circ} / \mathrm{s}$.

The means and standard deviations for the breakpoint of VORS are given in table 2. There was considerable variation in performance between individuals. Comparison of the means of maximum velocity of smooth pursuit and of the velocity for breakpoint of VORS shows a close relationship between the two. Although the variance associated with the pursuit and suppression data was high for the whole sample in each individual they were closely correlated. Therefore the criteria for abnormality should be a disproportion in performance between the pursuit and VORS as well as a reduction in performance in comparison with the normative data.

The results are presented in fig 1 which is a threedimensional representation of the means of pursuit velocity as a function of target velocity and frequency. On the same graph a "wedge" has been constructed to represent the means of the velocity breakpoints of VORS plotted as a function of frequency. The highest

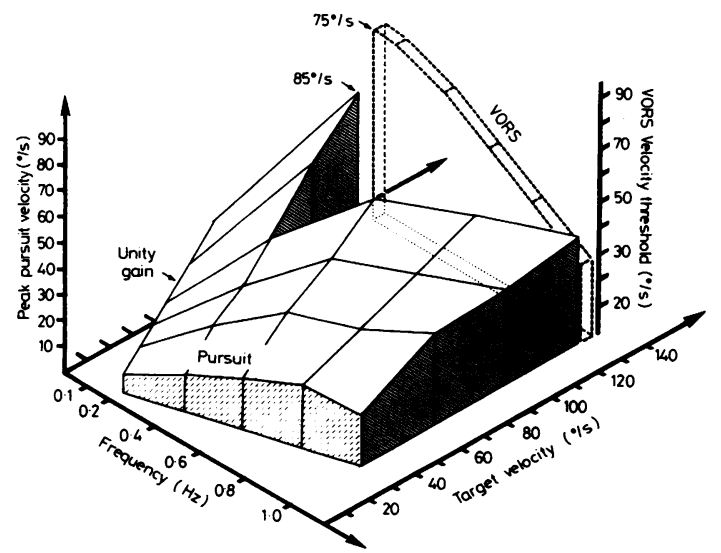

Fig 1 Three dimensional representation of the mean normative data for pursuit. Maximum pursuit velocities are expressed as a function of frequency and velocity of target motion. Superimposed is a "wedge" representing breakpoint for VORS at the same frequencies. values of the surfaces indicate a close relationship between the maximum velocity of pursuit which was attained at each frequency and the velocity of VORS breakdown. The Pearson correlation coefficient calculated on the averaged data between maximum pursuit velocity and VORS breakpoint was 0.96 , significant at between the $5 \%$ and $2 \%$ levels. The linear regression of VORS breakpoint velocity on maximum pursuit velocity as the independent variable gave a slope of $0 \cdot 83$.

The reason for constructing a 3-D display of the pursuit and suppression data is that if one adopts a simplistic view that performance is represented by the size and shape of the constructions then patterns of abnormality which may involve both frequency and velocity parameters show up clearly as abnormal shapes.

\section{B PATIENTS}

Many patients with a variety of neurological disorders, both supra and infra-tentorial, were examined clinically. Twenty of these with disordered pursuit and/or VORS were studied in detail, until the following three patterns of response that had been anticipated were encountered.

\section{Comparable impairment of pursuit and VORS:}

This was the most commonly observed pattern, encountered in patients with a wide spectrum of disorders, including multiple sclerosis, stroke, tumour, Parkinson's disease and sporadic and heredo-familial degenerative conditions. Fig 2 is a graphical representation of measurements from one patient. The pursuit "surface" indicates that pursuit movements were observed only at low frequencies, and that the maximum velocities of pursuit at these frequencies

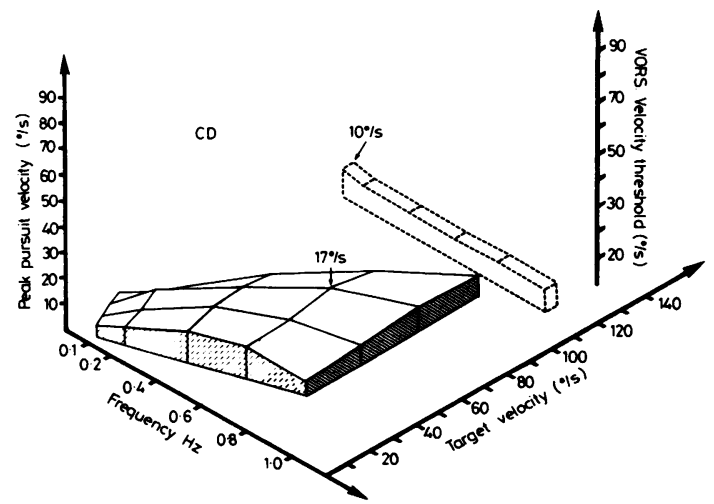

Fig 2 Pursuit as a function of frequency and velocity of target motion and VORS at the same frequencies, in a patient with pan-floccular degeneration. 
were well below normal. For example, at $0.2 \mathrm{~Hz}$ the maximum observed pursuit velocity was only $15^{\circ} / \mathrm{s}$. Similarly the breakpoints for VORS were abnormal and the thresholds conformed closely to the maximum observed pursuit velocities. At $0.2 \mathrm{~Hz}$ the breakpoint for VORS was $9^{\circ} / \mathrm{s}$. The patient was a 62-year-old male who presented with cold feet and pins and needles in the extremities. He had no other symptoms apart from blurred vision during head movement. The following oculomotor signs were the only neurological findings. There was gaze evoked nystagmus on looking left, right, and upwards, and rebound nystagmus was seen on recentering from the left, right, and downward eccentric positions. His inability to read a Snellen chart whilst head and shoulders were manually shaken indicated an abnormal gain of the VOR at higher frequency, subsequently confirmed by rotational stopping stimuli. In addition optokinetic nystagmus was grossly deranged. Radiological investigations were normal and the presumptive diagnosis was panfloccular degeneration of uncertain cause.

\section{Normal pursuit and impaired VORS:}

This was an uncommon finding encountered in a subject whose only symptom was an increased susceptibility to motion sickness, and in three patients with cerebellar pathology. Fig 3 illustrates the responses from one of the patients. Pursuit velocities were comparable to those of normal subjects through the range of frequencies tested, whilst the breakpoints for VORS at the same frequencies were depressed. For example-at $0.4 \mathrm{~Hz}$ the maximum pursuit velocity obtained was $74 \%$ s. but the break point for VORS was $10^{\circ} / \mathrm{s}$. This patient was a 23 -yearold woman with progressive spino-cerebellar degeneration associated with coeliac disease, kypho-

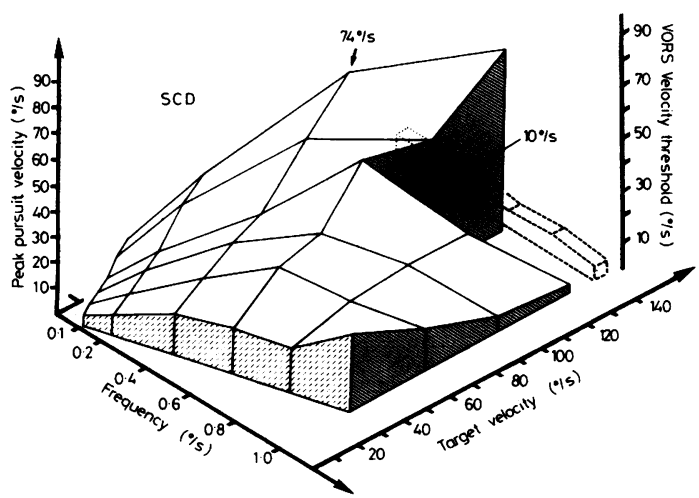

Fig 3 Pursuit as a function of frequency and velocity of target motion, and VORS at the same frequencies, in a patient with spino-cerebellar degeneration. scoliosis and features of Marfan's syndrome. Neurological signs were bilateral ptosis, bilateral optic atrophy, cerebellar ataxia, extensor plantars, areflexia and some sensory abnormalities. Neurootological examination revealed square wave jerks but no nystagmus. Full-field optokinetic stimulation produced normal optokinetic nystagmus whilst responses to impulsive rotational stopping stimuli were also normal. The square wave jerks created the illusion that pursuit was broken but recordings revealed smooth pursuit with square wave jerks superimposed. The second patient with the same pattern of response was her brother who had an almost identical neurological illness. The third patient was a diabetic with cerebellar atrophy confined to vermis and flocculo-nodular lobe, thought to be secondary to repeated hypoglycaemic episodes.

\section{Impaired pursuit and normal VORS:}

The third pattern was observed in only two patients. Results from one of these patients are represented in fig 4. Pursuit movements were generated only at low frequency and the maximum pursuit velocities achieved were low, for example only $16^{\circ} / \mathrm{s}$ at $0.4 \mathrm{~Hz}$. However, breakpoints for VORS were normal $\left(65^{\circ} / \mathrm{s}\right.$ at $0.4 \mathrm{~Hz}$ ) including those for frequencies equal to and greater than $0.6 \mathrm{~Hz}$, frequencies at which there was no recognisable pursuit. This patient was a 64 -yearold alcoholic, with a history of head trauma and symptoms of brain stem ischaemia secondary to postural hypotension. Examination revealed some impairment of cognitive functions, dysarthria and loss of dexterity of fine hand and finger movements.

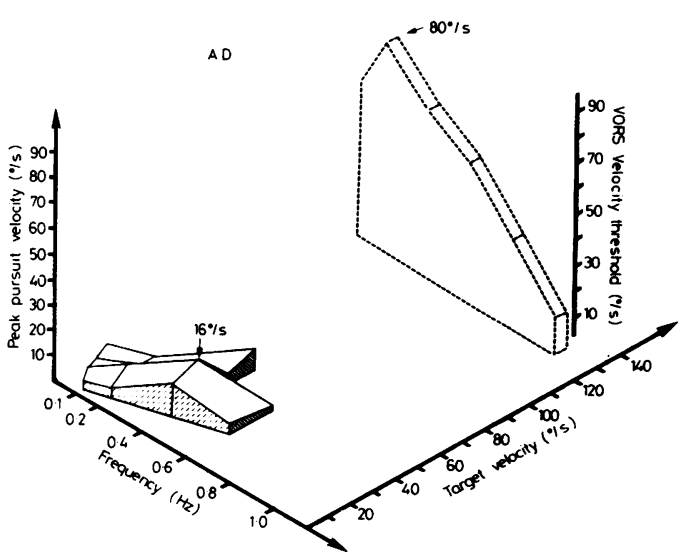

Fig 4 Pursuit as a function of frequency and velocity of target motion, and VORS at the same frequencies, in an alcoholic patient with a history of head trauma and recurrent brain stem ischaemia. 
There was no spontaneous nystagmus but in darkness there was $2 \mathrm{nd}^{\circ}$ nystagmus to the right and $1 \mathrm{st}^{\circ}$ nystagmus to the left. The other patient with the same pattern of response was a 72-year-old man with a history of vertebro-basilar ischaemia and transient global amnesia who was admitted with brain stem infarction.

\section{Discussion}

The data from normal subjects in our examination are in agreement with previous findings in that they show a direct relationship between the performance of smooth pursuit and VORS. ${ }^{\text {The }}$ Thesent results extend the previous observations by testing the dynamic range of pursuit as a function of frequency and velocity of target motion. It is the upper limit of velocity of pursuit at each frequency that corresponds best to the velocity at which VORS breaks down.

Previous studies of visual-vestibular interaction have tested the performance of the smooth pursuit reflex by using constant velocity stimuli and have expressed the performance of pursuit in terms of gain. The present experiments show that increasing the amplitude of target excursion can push up the velocity of pursuit to very high levels indicating that although there may be an eventual upper limit to the maximum velocity of which the pursuit system is capable, the determining factor, in practicable terms, is an acceleration limit. This finding is in accordance with recent data on the performance of pursuit in men and monkeys, showing that pursuit gain is linearly related to target acceleration. ${ }^{6}$ These findings indicate that when testing pursuit in relation to VORS the correct procedure to adopt is to determine the upper limit of velocity at each test frequency. This method will yield more relevant information than measurements of gain at standard target velocities.

In agreement with previous authors 23 it was found that the majority of patients with broken pursuit had a comparable impairment of VORS. This was a graded phenomenon with deficits from mild to severe and was found in a wide spectrum of disorders involving both infra- and supratentorial lesions. Of particular interest is the corresponding degradation of pursuit and suppression in patients with lesions affecting corticofugal pathways from the visual cortex (one patient with parietal glioma). Diffuse tumours in the parietal subcortex may cause pursuit defects in patients with normal acuity and full fields. The relationship between pursuit and VORS in these patients strongly suggests that some common information relating to the movement of visual images used for the generation of pursuit and visually mediated VORS.

The second pattern of response in which pursuit was relatively intact and VORS almost absent was found in patients with cerebellar lesions and in a "volunteer" with increased susceptibility to motion sickness. Unfortunately none of the patients provided a precise anatomical location of the lesion. However the observations are of significance for pathophysiology. It has been established that in primates an intact flocculo-nodular lobe is essential for the preservation of smooth pursuit and VORS. 78 The finding that in cerebellar lesions pursuit may be intact whilst VORS is impaired indicates that within the cerebellum there must be a division of pursuit and VORS functions and that the loci responsible for VORS are not on the main pursuit pathway at that level. The finding of intact pursuit and disordered VORS has the further implication that although suppression may be derived from something akin to a pursuit signal it does not rely on an algebraic cancellation of the two at the summing junction of the final common pathway. One might expect that patients with disordered pursuit may be able to suppress their VOR by non-visual means because non-visual suppression has been amply demonstrated in normal subjects. ${ }^{10}$ This is achieved by asking the subject to imagine a target moving with the head during rotation and predict the motion stimulus. It is likely that these signals are channelled through the same pathways as those mediating visual VORS, because our patients demonstrated no capacity to suppress in this way either. Recent models of VORS would concur with this interpretation. 1011

There are several possible explanations for the third pattern of response in which pursuit was highly deranged and VORS was normal. The patients with this pattern had vascular brain stem lesions and in addition there was clinical evidence of diffuse cerebral dysfunction. The first explanation is that the corticofugal pathways mediating both pursuit and VORS were intact down to the lower level of common anatomical and functional organisation, and thereafter pursuit pathways were alone disrupted. Secondly, it might be possible that there was an apraxia of pursuit but not VORS resulting from cerebral cortical dysfunction. Thirdly, it is possible that these patients, despite their neurological disorders, retained some ability for non-visually mediated VORS.

One subject who was asymptomatic, except for an unusual susceptibility to motion sickness, had highly abnormal VORS. His smooth pursuit was normal. However, VORS broke down at $25^{\circ}$ /s even at low frequencies. When travelling in a coach or the rear seat of a car, most of the visual environment moves with the subject and consequently a person with poor VORS would experience unstable vision and this factor could be significant in the aetiology of motion sickness in otherwise normal subjects. 
Our findings confirm and systematise the anecdotal observations that smooth pursuit performance and VORS may become dissociated in patients with neurological disorders, although in general both are comparably deranged. The only other study in which dissociations between pursuit and VORS were found and quantified is that of Mira $e^{2}$ all ${ }^{12}$ who concluded that impaired VORS did not correlate with smooth pursuit defects in patients with cerebellar disorders, implying that all cerebellar patients have a dissociation between the two.However, of the patients with cerebellar disorders tested in the present study, the majority showed comparable decrement of pursuit and VORS, and only three showed dissociation. From physiological considerations this must necessarily be the case, for the same area of the cerebellum, namely the flocculus, is intimately concerned with both functions. ${ }^{78}$

In conclusion the testing of smooth pursuit and VORS is clinically useful, for a defect of either is unequivocal evidence for central lesions. The finding that VORS may be impaired in the absence of a defect of smooth pursuit has the additional implication that clinical testing of VORS would be a useful addition to routine clinical neurological examination. Generally, in patients with neurological disorders, abnormal pursuit and impaired VORS have no localising value with the possible exception of the unusual case in which pursuit is normal and VORS reduced. Our experience suggests that this indicates a cerebellar lesion.

\section{References}

${ }^{1}$ Barnes GR, Benson AJ, Prior ARJ. Visual-vestibular interaction in the control of eye movement. Aviat Space Environ Med 1978;49:557-64.

2 Dichgans J, Von Reutern GM, Römmelt U. Impaired suppression of vestibular nystagmus by fixation in cerebellar and non-cerebellar patients. Arch Psychiatr Nervenkr 1978; 226: 183-99.

3 Halmagyi GM, Gresty MA. Clinical signs of visualvestibular interaction. J Neurol Neurosurg Psychiatry 1979;42:934-9.

${ }^{4}$ Lennerstrand G, Zee DS, Keller EL. Functional Basis of Ocular Motility Disorders Chapter "Disorders of EyeHead Coordination." Oxford, Pergamon 1982. 418-9.

5 Chambers BR, Gresty MA. Effects of fixation and optokinetic stimulation on vestibulo-ocular reflex suppression. J Neurol Neurosurg Psychiatry 1982;45:998-1004.

${ }^{6}$ Lisberger SG, Evinger C, Johanson GW, Fuchs AF. Relationship between eye acceleration and retinal image velocity during foveal smooth pursuit in man and monkey. J Neurophysiol 1981;46:229-49.

7 Takemori S, Cohen B. Loss of visual suppression of vestibular nystagmus after floccular lesions Brain Res 1974;72:213-24.

8 Zee DS, Yamazaki A, Gucer G. Ocular motor abnormalities in trained monkeys with floccular lesions Society for Neuroscience Abstracts 1978;4:168.

9 Barr CC, Schultheis LW, Robinson DA. Voluntary, nonvisual control of the human vestibulo-ocular reflex. Acta Otolaryngol 1976;81:365-75.

10 Schmid R, Zambarbieri D, Magenes G. Modifications of vestibular nystagmus produced by fixation of visual and non-visual targets. Ann NY Acad Sci 1981;374:689705.

11 Robinson DA. Model of vestibulc-ocular reflex suppression in: Lennerstrand G, Zee DS, Keller EL, eds. In Press. Oxford, Pergamon Press 1982.

12 Mira E, Mevio E, Zanocco P, Castlenuovo, P. Impaired suppression of vestibular nystagmus by fixation of visual and acoustic targets in neurological patients. Ann NY Acad Sci 1981;374:706-21. 